DOI: $10.17516 / 1997-1397-2020-13-4-503-514$

УДК $517.55+517.33$

\title{
On New Decomposition Theorems in some Analytic Function Spaces in Bounded Pseudoconvex Domains
}

\author{
Romi F. Shamoyan* \\ Elena B. Tomashevskaya ${ }^{\dagger}$ \\ Bryansk State University \\ Bryansk, Russian Federation
}

Received 06.04.2020, received in revised form 23.05.2020, accepted 06.07.2020

\begin{abstract}
We provide new sharp decomposition theorems for multifunctional Bergman spaces in the unit ball and bounded pseudoconvex domains with smooth boundary expanding known results from the unit ball.

Namely we prove that $\prod_{j=1}^{m}\left\|f_{j}\right\|_{X_{j}} \asymp\left\|f_{1} \ldots f_{m}\right\|_{A_{\alpha}^{p}}$ for various $\left(X_{j}\right)$ spaces of analytic functions in bounded pseudoconvex domains with smooth boundary where $f, f_{j}, j=1, \ldots, m$ are analytic functions and where $A_{\alpha}^{p}, 0<p<\infty, \alpha>-1$ is a Bergman space. This in particular also extend in various directions a known theorem on atomic decomposition of Bergman $A_{\alpha}^{p}$ spaces.

Keywords: pseudoconvex domains, unit ball, Bergman spaces, decomposition theorems, Hardy type spaces.

Citation: R.F. Shamoyan, E.B. Tomashevskaya, On New Decomposition Theorems in some Analytic Function Spaces in Bounded Pseudoconvex Domains, J. Sib. Fed. Univ. Math. Phys., 2020, 13(4), 503-514. DOI: 10.17516/1997-1397-2020-13-4-503-514.
\end{abstract}

\section{Introduction and preliminaries}

The problem we consider is well-known for functional spaces in $\mathbb{R}^{n}$ (the problem of equivalent norms) (see, for example, [1]).

Let $X,\left(X_{j}\right)$ be a function space in a fixed domain $D$ in $\mathbb{C}^{n}$ (normed or quazinormed) we wish to find equivalent expression for $\left\|f_{1} \ldots f_{m}\right\|_{X} ; m \in \mathbb{N}$. Note these are closely connected with spaces on product domains since

$$
f\left(z_{1}, \ldots, z_{m}\right)=\prod_{j=1}^{m} f_{j}\left(z_{j}\right), \quad\|f\|_{X}=\prod_{j=1}^{m}\left\|f_{j}\right\|_{X_{j}}, \quad z_{j} \in D ; j=1, \ldots, m .
$$

These our results also extend some well-known assertions on atomic decomposition of Bergman $A_{\alpha}^{p}$ type spaces as we will see below. For $m=1$ Hardy space case (see, for example, $[2-4])$.

To study such group of functions it is natural, for example, to ask about structure of each $\left\{f_{j}\right\}_{j=1}^{m}$ of this group.

This can be done for example if we turn to the following question find conditions on $\left\{f_{1}, \ldots, f_{m}\right\}$, so that $\left\|f_{1}, \ldots, f_{m}\right\|_{X} \asymp \prod_{j=1}^{m}\left\|f_{j}\right\|_{X_{j}}$ sharp $(R)$ decomposition is valid. In this case for example we have if for some positive constant $c$;

\footnotetext{
*rsham@mail.ru

†tomele@mail.ru

(C) Siberian Federal University. All rights reserved
} 


$$
\prod_{j=1}^{m}\left\|f_{j}\right\|_{X_{j}} \leqslant c\left\|f_{1}, \ldots, f_{m}\right\|_{X}
$$

then we have each $f_{j}, f_{j} \in X_{j} ; j=1, \ldots, m$, where $X_{j}$ is a new normed (or quazinormed) function space in $D$ domain and we can easily now provide properties of $f_{j}$ based on facts of already known one functional function space theory. (For example to use known theorems for each $f_{j} \in X_{j} ; j=1, \ldots, m$ on atomic decompositions). This idea was used for Bergman spaces in the unit ball then in bounded pseudoconvex domains with smooth boundary in recent papers [5] and [6]. In this paper we extend these results in various directions using modification of known proof.

We refer to $[5,6]$ for a complete and not difficult proof of a basic known "purely $A_{\alpha}^{p}$ " case then in this paper show in details how to modify it to get new results. The old known proof is simple and very flexible as it turns out and we can easily get, as we can see below, various new interesting results from it directly. This remark is leading us to provide only some sketchy arguments sometimes below of proofs when we deal with new theorems, since the core of all proofs is basically the same in all our theorems. Here is partially the transparent proof of the classical case of the Bergman space $A_{\alpha}^{p}$ case in the unit ball $B_{n}$ of $\mathbb{C}^{n}$. The case of $A_{\alpha}^{p}$ Bergman space in more general bounded pseudoconvex domain can be seen in our recent paper [6].

We define $A_{\alpha}^{p}$ space as usual

$$
A_{\alpha}^{p}=\left\{f \in H(B):\|f\|_{A_{\alpha}^{p}}^{p}=\int_{B}|f(z)|^{p}(1-|z|)^{\alpha} d v(z)<\infty\right\},
$$

$d v$ is a Lebeques measure on $B, f_{j}$ is analytic in $B, 0<p<\infty, \alpha>-1, j=1, \ldots, m$ and where $H(B)$ is a class of all analytic functions in the unit ball $B$.

We see in [6] that $\left\|f_{1} \ldots f_{m}\right\|_{A_{\tau}^{p}} \asymp \prod_{j=1}^{m}\left\|f_{j}\right\|_{A_{\alpha}^{p}}$ is valid under certain integral (A) condition if $p \leqslant 1$ and if $\tau=\tau\left(p, \alpha_{1}, \ldots, \alpha_{m}, m\right)$.

We denote constants as usual by $C, C_{1}, C_{2}, \ldots$

Note from our discussion above the only interesting part is to show that

$$
\prod_{j=1}^{m}\left\|f_{j}\right\|_{A_{\alpha_{j}}^{p}\left(B_{n}\right)} \leqslant c_{1}\left\|f_{1} \ldots f_{m}\right\|_{A_{\tau}^{p}\left(B_{n}\right)}
$$

since the reverse follows directly from the uniform estimate (see $[6,7])$.

$$
|f(z)|(1-|z|)^{\frac{\alpha_{j}+n+1}{p}} \leqslant c\|f\|_{A_{\alpha_{j}}^{p}} ; 0<p<\infty, \alpha_{j}>-1, j=1, \ldots, m
$$

and ordinary induction. This also lead easily to the fact that $\tau$ can be calculated

$$
\tau=(n+1)(m-1)+\sum_{j=1}^{m} \alpha_{j} ; \alpha_{j}>-1 ; 0<p<\infty .
$$

Note similar very simple proof based only on various known uniform estimates can be used in all our proofs below in similar inequalities for various spaces. So we mainly concentrate on reverse to $(S)$ estimates. Let further $H^{p}$ be a usual Hardy $H^{p}$ space in $B_{n}$ (see $[7,8]$ ).

Note further if $\alpha_{0}$ is large enough and if

$$
\prod_{i=1}^{m} f_{i}\left(\omega_{i}\right)=c_{\alpha} \int_{B} \frac{\prod_{i=1}^{m} f_{i}(z)(1-|z|)^{\alpha} d v(z)}{\prod_{i=1}^{m}\left(1-\tilde{z} \omega_{i}\right)^{\frac{n+1+\alpha}{m}}}, \quad \omega_{j} \in B, j=1, \ldots, m, \alpha>\alpha_{0},
$$


then we have easily using directly well-known estimates (see [7]) from last equality for $p \leqslant 1$ (we refer to [6] for details in more general situation).

$$
\begin{gathered}
I=\prod_{i=1}^{m} \int_{B}\left|f_{k}\left(z_{k}\right)\right|^{p}\left(1-\left|z_{k}\right|^{2}\right)^{\alpha_{k}} d v\left(z_{k}\right)=\int_{B} \ldots \int_{B} \prod_{k=1}^{m}\left|f_{k}\left(z_{k}\right)\right|^{p} \cdot \prod_{k=1}^{m}\left(1-\left|z_{k}\right|^{2}\right)^{\alpha_{k}} \cdot \prod_{k=1}^{m} d v\left(z_{k}\right) \leqslant \\
\leqslant c \int_{B} \prod_{k=1}^{m}\left|f_{k}(z)\right|^{p}\left(1-\left|z_{k}\right|^{2}\right)^{\tau} d v(z) \times \int_{B} \ldots \int_{B} \frac{\prod_{k=1}^{m}\left(1-\left|z_{k}\right|^{2}\right)^{\alpha_{k}} \prod_{k=1}^{m} d v\left(z_{k}\right)}{\prod_{k=1}^{m}\left|1-\tilde{z} z_{k}\right|^{\left(\frac{n+1+\alpha}{m}\right) p}} ;
\end{gathered}
$$

where $\alpha_{k}>-1, k=1, \ldots, m, \tau=(n+1+\alpha) p-(n+1)>-1, \alpha>\alpha_{0}=\alpha_{0}(p, \vec{\alpha}, n, m)$. And hence we have finnally

$$
c_{1} \int_{B} \prod_{k=1}^{m}\left|f_{k}(z)\right|^{p}\left(1-\left|z_{k}\right|^{2}\right)^{\tau_{1}} d v(z)<\infty,
$$

where $0<p \leqslant 1 ; \tau_{1}=(m-1)(n+1)+\sum_{k=1}^{m} \alpha_{k}$; and $\alpha_{k}>-1, \tau_{1}>-1, \alpha>\alpha_{0}$.

This result is valid also for $p>1$ (see [5]). We will repeat this type simple argument several times below.

The same more general problem which we consider in bounded pseudoconvex domain $D$ is the following. To find equivalent expressions for $\left\|f_{1} \ldots f_{m}\right\|_{X} ; f_{j} \in H(D), j=1, \ldots, m$. Can we also say that each $f i$ can be decomposed into "atoms" (BMOA atoms, Bloch atoms, Hardy atoms, Bergman atoms (see $[2-4,6,7,9,10])$ ) if

$$
\int_{D}\left|\prod_{j=1}^{m} f_{j}(z)\right|^{p} \delta^{\tau}(z) d v(z)<\infty, \quad 0<p<\infty, \quad \tau>-1 ; \quad \delta(z)=\operatorname{dist}(z, \partial D)
$$

and $d v$ is a Lebeques measure on $D$. Only for $m=1 A_{\alpha}^{p}$ Bergman class the answer is well known in the unit ball and in bounded pseudoconvex domains (see $[5-7,11]$ ).

For $m>1$ the answer is known only partially each $\left(f_{j}\right)$ can be decomposed into $A_{\alpha_{j}}^{p}$ atoms for some $\alpha_{j}$ see $[5,6]$. For $m=1$ Hardy space and other spaces (see [2-4,7]) and references there.

We extend these known results in various directions below. It is easy to note that in our proof at least one $f_{j}$ must be decomposed into $A_{\alpha}^{p}$ atoms.

Let us remark the following typical for this paper fact in bounded pseudoconvex $D$ domains an extension of a classical result namely the following result is valid (note same result with the same proof even can be provided with the same proofs in unbounded tube domains over symmetric cones). This will be studied in our next papers. Let $H^{p}$ and $A_{\alpha}^{p}, 0<p<\infty, \alpha>-1$ be Bergman and Hardy space in $D$ domain (see $[6,8,12,13]$ ) and definitions below.

Note since proofs are rather simple some arguments have sketchy forms and can be easily recovered by readers $($ see $[6,13])$.

We denote by $C_{\beta}$ Bergman representation constant below.

Theorem 1. Let $f_{i} \in A_{\alpha_{i}}^{p_{i}}, i=1, \ldots, k ; f_{i} \in H^{p_{i}} ; i=k+1, \ldots, m, p_{i} \leqslant 1, i=1, \ldots, m$, $\alpha_{j}>-1, j=1, \ldots, k, \tau=n(m-k)+(n+1)(k-1)+\sum_{j=1}^{k} \alpha_{j}$, then

$$
\int_{D} \prod_{j=1}^{m}\left|f_{j}\right|^{p_{j}} \delta(z)^{n(m-k)+\left(\sum_{j=1}^{k} \alpha_{j}\right)+(n+1)(k-1)} d v(z) \leqslant C \prod_{j=k+1}^{m}\left\|f_{i}\right\|_{H^{p_{i}}}^{p_{i}} \prod_{j=1}^{k}\left\|f_{i}\right\|_{A_{\alpha_{i}}^{p_{i}}}^{p_{i}}
$$

and for cases when $p_{i}=p, j=1, \ldots, m$ the reverse is also true and we have a new sharp result 
$I(\vec{f})=\int_{D} \prod_{j=1}^{m}\left|f_{j}(z)\right|^{p} \delta(z)^{n(m-k)+\left(\sum_{j=1}^{k} \alpha_{j}\right)+(n+1)(k-1)} d v(z) \asymp \prod_{j=k+1}^{m}\left\|f_{i}\right\|_{H^{p}}^{p} \prod_{j=1}^{k}\left\|f_{j}\right\|_{A_{\alpha_{j}}^{p}}^{p} ;$

if

$$
\begin{aligned}
& \prod_{j=1}^{m} f_{i}\left(w_{i}\right)=C_{\beta} \int_{D}\left(\prod_{j=1}^{m} f_{j}(z)\right) \times \prod_{j=1}^{m} K_{\frac{\beta+n+1}{m}}\left(z, w_{j}\right) \delta^{\beta}(w) d v(w) ; \\
& \beta>\beta_{0}, w_{j} \in D_{j}, j=1, \ldots, k \\
& w_{j} \in D, j=k+1, \ldots, m .
\end{aligned}
$$

This Theorem 1 is probably true also for $p_{i}>1$ (see [5,6] for proof in this case based only on Holder inequality) we give also very similar same type result for analytic $A_{\alpha}^{\infty, p}$ weighted Hardy class below.

\section{Remark 1.}

1) Note for $m=1(T)$ integral condition vanishes (see [12]) and we have an obvious relation. and hence $f_{1}$ can be decomposed into atoms, $f_{1} \in A_{\alpha}^{p}$ (see $\left.[6,7]\right)$.

2) Our result as a root has the following simple estimate in the unit disk which can be easily checked.

$$
\int_{U} \prod_{i=1}^{k}\left|f_{i}(z)\right|^{p_{i}} \delta(z)^{k-1} d v(z) \leqslant C \prod_{i=1}^{k}\left\|f_{i}\right\|_{H^{p_{i}}}^{p_{i}}, \quad p_{i} \in(0, \infty), f_{i} \in H^{p_{i}}, i=1, \ldots, k, k \in \mathbb{N} .
$$

\section{Remark 2.}

Note for $m>1$ we can hence using $(\widetilde{\widetilde{A}})$ decompose if $I(\vec{f})<\infty$ each function $\left(f_{j}\right)$ to $H^{p}$ atoms and (or) $A_{\alpha}^{p}$ atoms using well-known one functional results. Note for $m=1(T)$ vanish and we obtain $A_{\alpha}^{p}$ atomic decomposition classical result.

We refer to [14] for other new interesting sharp results in mulifunctional Bergman spaces.

\section{Main results}

We provide our main results in this section. Throughout this paper $H(D)$ denotes the space of all holomorphic functions on an open set $D \subset \mathbb{C}^{n}$.

We follow notation from [11]. Let $D$ be a bounded strictly pseudoconvex domain in $\mathbb{C}^{n}$ with smooth boundary, let $d(z)=\operatorname{dist}(z, \partial D)$.

Then there is a neighborhood $U$ of $\bar{D}$ and $\rho \in C^{\infty}(U)$ such that $D=\{z \in U: \rho(z)>0\}$, $|\nabla \rho(z)| \geqslant c>0$ for $z \in \partial D, 0<\rho(z)<1$ for $z \in D$ and $-\rho$ is strictly plurisubharmonic in a neighborhood $U_{0}$ of $\partial D$. Note that $d(z) \asymp \rho(z), z \in D$. Then there is an $r_{0}>0$ such that the domains $D_{r}=\{z \in D: \rho(z)>r\}$ are also smoothly bounded strictly pseudoconvex domains for all $0 \geqslant r \geqslant r_{0}$. Let $d \sigma_{r}$ be the normalized surface measure on $\partial D_{r}$ and $d v$ the Lebesgue measure on $D$. The following mixed norm spaces were investigated in [11]. For $0<p<\infty$, $0<q \leqslant \infty, \delta>0$ and $k=0,1,2, \ldots$ set

$$
\|f\|_{p, q, \delta ; k}=\left(\sum_{|\alpha| \leqslant k} \int_{0}^{r_{0}}\left(r^{\delta} \int_{\partial D_{r}}\left|D^{\alpha} f\right|^{p} d \sigma_{r}\right)^{q / p} \frac{d r}{r}\right)^{1 / q}, 0<q<\infty
$$

and weighted Hardy space $\left(A_{0}^{p, \infty}=H^{p}\right)$

$$
\|f\|_{p, \infty, \delta ; k}=\sup _{0<r<r_{0}} \sum_{|\alpha| \leqslant k}\left(r^{\delta} \int_{\partial D_{r}}\left|D^{\alpha} f\right|^{p} d \sigma_{r}\right)^{1 / q}, 0<q<\infty
$$


where $D^{\alpha}$ is a derivative of $f$ (see [11]) The corresponding spaces $A_{\delta ; k}^{p, q}=A_{\delta ; k}^{p, q}(D)=\{f \in H(D)$ : $\left.\|f\|_{p, q, \delta ; k<\infty}\right\}$ are complete quasi normed spaces, for $p, q \geqslant 1$ they are Banach spaces. We mostly deal with the case $k=0$, when we write simply $A_{\delta}^{p, q}$ and $\|f\|_{p, q, \delta}$. We also consider this spaces for $p=\infty$ and $k=0$, the corresponding space is denoted by $A_{\delta}^{\infty, p}=A_{\delta}^{\infty, p}(D)$ and consists of all $f \in H(D)$ such that

$$
\|f\|_{\infty, p, \delta}=\left(\int_{0}^{r_{0}}\left(\sup _{\partial D_{r}}|f|\right)^{p} r^{\delta p-1} d r\right)^{1 / p}<\infty .
$$

Also, for $\delta>-1$, the space $A_{\delta}^{\infty}=A_{\delta}^{\infty}(D)$ consist of all $f \in H(D)$ such that

$$
\|f\|_{A_{\delta}^{\infty}}=\sup _{z \in D}|f(z)| \rho(z)^{\delta}<\infty
$$

and the weighted Bergman space $A_{\delta}^{p}=A_{\delta}^{p}(D)=A_{\delta+1}^{p, p}(D)$ consists of all $f \in H(D)$ such that

$$
\|f\|_{A_{\delta}^{p}}=\left(\int_{D}|f(z)|^{p} \rho^{\delta}(z) d v(z)\right)^{1 / p}<\infty
$$

We denote by $K_{\beta}$ the weighted Bergman kernel on $D$ (see $\left.[6,12]\right)$.

Since $|f(z)|^{p}$ is subharmonic (even plurisubharmonic) for a holomorphic $f$, we have $A_{s}^{p}(D) \subset$ $A_{t}^{\infty}(D)$ for $0<p<\infty, s p>n$ and $t=s$. Also, $A_{s}^{p}(D) \subset A_{s}^{1}(D)$ for $0<p \leqslant 1$ and $A_{s}^{p}(D) \subset$ $A_{t}^{1}(D)$ for $p>1$ and $t$ sufficiently large. Therefore we have an integral representation

$$
f(z)=C_{\beta} \int_{D} f(\xi) K(z, \xi) \rho^{t}(\xi) d v(\xi), \quad f \in A_{t}^{1}(D), z \in D,
$$

where $K(z, \xi)$ is a kernel of type $t$, that is a smooth function on $D \times D$ such that $|K(z, \xi)| \leqslant$ $C|\tilde{\Phi}(z, \xi)|^{-(n+1+t)}$, where $\tilde{\Phi}(z, \xi)$ is so called Henkin-Ramirez function for $D$. Note that $\left(^{*}\right)$ holds for functions in any space $X$ that embeds into $A_{t}^{1}$. We review some facts on $\tilde{\Phi}$ and refer reader to [15] for details. This function is $C^{\infty}$ in $U \times U$, where $U$ is a neighborhood of $\bar{D}$, it is holomorphic in $z$, and $\tilde{\Phi}(\zeta, \zeta)=\rho(\zeta)$ for $\zeta \in U$. Moreover, on $\bar{D} \times \bar{D}$ it vanishes only on the diagonal $(\zeta, \zeta), \zeta \in \partial D$. Locally, it is up to a non vanishing smooth multiplicative factor equal to the Levi polynomial of $\rho$. From now on the work with a fixed Henkin-Ramirez function $\widetilde{\Phi}$.

The proof of the following theorem is very similar to the proof of the Theorem 1.

Theorem 2. Let $f_{i} \in A_{\beta_{i}}^{\infty}, i=1, \ldots, k$ and $f_{i} \in A_{\alpha_{i}}^{p_{i}}, i=k+1, \ldots, m$.

Let $\beta_{j} \geqslant 0, j=1, \ldots, m$, let also $p_{i} \leqslant 1$, let $\alpha_{j}>-1 ; j=1, \ldots, m$; then we have

$$
\int_{D} \prod_{j=1}^{m}\left|f_{j}\right|^{p_{i}} \cdot \delta(z)^{\sum_{j=1}^{k}\left(\beta_{j} p_{j}\right)+(n+1)(m-k-1)+\sum_{i=1}^{m} \alpha_{i}} d v(z) \leqslant C \prod_{i=k+1}^{m}\left\|f_{i}\right\|_{A_{\alpha_{i}}^{p_{i}}}^{p_{i}} \times \prod_{i=1}^{k}\left\|f_{i}\right\|_{A_{\beta_{i}}^{\infty}}^{p_{i}}
$$

and if $p_{i}=p, i=1, \ldots, m$ we have a sharp result (the reverse of $(K)$ is valid) if

$$
\prod_{i=1}^{m} f_{i}\left(w_{i}\right)=C_{\beta} \int_{D} \prod_{j=1}^{m} f_{j}(z) \times K_{\frac{\beta+n+1}{m}}\left(z, w_{j}\right) \delta^{\beta}(z) d v(z) ; \beta>\beta_{0} ; w_{j} \in D, j=1, \ldots, m .
$$

The same type results with very similar proof is valid not only for $A_{\beta}^{\infty}$ but also for weighted Hardy space

$$
A_{\alpha}^{p, \infty}=\left\{f \in H(D): \sup _{\varepsilon>0}\left(\int_{\partial D_{\varepsilon}}|f(\xi)|^{p} \tilde{\sigma}(\xi)\right)^{\frac{1}{p}} \times \varepsilon^{\alpha}<\infty ; \alpha \geqslant 0 ; 0<p<\infty\right\}
$$

where $\partial D_{\varepsilon}=\{z: \rho(z)=\varepsilon\}, \tilde{\sigma}(\xi)$ is a Lebeques measure on $\partial D_{\varepsilon}$ (see [11] for these analytic Hardy type spaces). 
Theorem 2 can be also viewed similarly (as Theorem 1) as another direct extension of a known theorem on atomic decomposition of classical Bergman space $A_{\alpha}^{p}$ in the $D$ domain. Indeed we can easily see that (see $[7,12]$ ) for $m=1$ in the ball (T) integral condition vanishes and we have $A_{\alpha}^{p}$ known atomic decomposition result. For $m>1$ taking into account known atomic decomposition theorems (see [9]) for $A_{\alpha}^{p}$ and $A_{\beta}^{\infty}$ in $D$, each $f_{j}, j=1, \ldots, m$ from Theorem 2 can be decomposed into $A_{\beta}^{\infty}$ or $A_{\alpha}^{p}$ atoms.

The same type result is valid for some Herz type spaces in bounded pseudoconvex domains and BMOA type spaces in the unit ball instead of $A_{\beta}^{\infty}$.

We refer to [16] for some interesting results in such type analytic function spaces.

Namely, let $B(z, r)$ be a Kobayashi ball in $D, z \in D, r>0$ (see [11]).

Let also $B_{\alpha}^{p q}, \tilde{B}_{\alpha}^{p q}, p, q \in(0, \infty), \alpha>-1$, be Herz type spaces in pseudoconvex $D$ domain

$$
\begin{aligned}
& B_{\alpha}^{p, q}(D)=\left\{f \in H(D): \int_{D}\left(\int_{B(z, r)}|f(w)|^{q} \delta^{\alpha}(w) d v(w)\right)^{\frac{p}{q}} d v(z)<\infty\right\}, \\
& \widetilde{B}_{\alpha}^{p, q}(D)=\left\{f \in H(D): \sum_{k \geqslant 0}\left(\int_{B\left(a_{k}, r\right)}|f(w)|^{q} \times \delta^{\alpha}(w) d v(w)\right)^{\frac{p}{q}}<\infty\right\},
\end{aligned}
$$

where $\left\{a_{k}\right\}$ is known $r$-lattice in $D$ (see [13]).

Let also

$$
B M O A_{s, \beta, t}^{p}\left(B_{n}\right)=\left\{f \in H\left(B_{n}\right): \sup _{w \in B} \int_{B} \frac{|f(z)|^{p} \times(1-|z|)^{s} d v(z)}{|1-\bar{w} z|^{\beta}}(1-|w|)^{t}<\infty\right\},
$$

be BMOA type space in the unit ball (see also [7,17,18]), where $0<p, q<\infty ; s>-1, \beta, t \geqslant 0$.

Uniform estimates for BMOA in the unit ball can be seen in [7], for $B_{\alpha}^{p, q}$ and $\widetilde{B}_{\alpha}^{p, q}$ Herz type spaces they can be easily obtained also based on elementary known estimates (see $[12,13]$ )

$$
|f(z)|^{p} \leqslant \widetilde{C}\left(\int_{B(z, r)}|f(w)|^{p} d v(w)\right) \cdot \delta^{-(n+1)}(z) ; z \in D, 0<p<\infty .
$$

As a result we immediately have that

$$
\int_{D} \prod_{k=1}^{m}\left|f_{k}\right|^{p_{i}} \times \delta(z)^{s} d v(z) \leqslant C \prod_{k=1}^{t}\left\|f_{k}\right\|_{A_{\beta_{k}}^{p_{k}}}^{p_{k}} \times \prod_{k=t+1}^{m}\left\|f_{k}\right\|_{B_{\alpha_{k}}^{\widetilde{q_{k}}}, \widetilde{\ell_{k}}}^{\widetilde{l_{k}}}
$$

for some $s=s(\vec{p}, n, m, \vec{\alpha}, \vec{\beta}, \overrightarrow{\widetilde{p}}, \overrightarrow{\widetilde{q}})$ and the same type estimate obviously is valid for $\widetilde{B}_{\alpha}^{p, q}(D)$ and $B M O A_{s, \beta, t}^{p}\left(B_{n}\right)$ (We simply replace $\prod_{k=t+1}^{m}\left\|f_{k}\right\|_{B^{\widetilde{p}_{k}, \widetilde{q}_{k}}}$ by quazinorms of these spaces).

For particular values of parameters we under integral condition ( $\mathrm{T}$ ) can again show similarly that this $\left(A_{3}\right)$ estimate is sharp, so each $f_{k}$ can be decomposed into BMOA and $\widetilde{B}_{\alpha}^{p, q}\left(B_{\alpha}^{p, q}\right)$ atoms if only $\prod_{i=1}^{m}\left|f_{i}\right|^{p} \in L_{s}^{1}(D), 0<p \leqslant 1$ for some $s$.

These results in details will be given in another our paper.

Proof of Theorem 1.

The $(\tilde{\tilde{A}})$ estimate follows from two known uniform estimates directly

$$
|f(z)|(1-|z|)^{\frac{n}{p_{i}}} \leqslant C\|f\|_{H^{p_{i}}}^{p_{i}}, z \in D
$$

and

$$
|f(z)|(1-|z|)^{\frac{n+1+\alpha_{i}}{p_{i}}} \leqslant C_{1}\|f\|_{A_{\alpha_{i}}^{p_{i}}}^{p_{i}}, z \in D, \alpha_{i}>-1,0<p<\infty
$$


(see $[6,7,11,13,19])$. The $\left(A_{3}\right)$ can be shown similarly. To get the reverse estimate we must use first that for $p \leqslant 1$ (see [6]) we have

$$
\left(\int_{D}|f(w)| \cdot \prod_{j=1}^{m}\left|K_{\tau}\left(z_{j}, w\right)\right| \delta^{\alpha}(w) d v(w)\right)^{p} \leqslant C \int_{D}|f(w)|^{p} \cdot\left|K_{\tau}(z, w)\right|^{p} \delta^{\alpha p+(n+1)(p-1)}(w) d v(w),
$$

$\tau>0, \alpha>-1, p \leqslant 1$ and also the following known lemma (see $[6,12,13,19,20]$ ). (Forelly-Rudin type estimates).

Lemma 1. Let $\tilde{\alpha}, \beta>-1, s>0, y \in D, 0<t<t_{0}=t_{0}(\lambda, r)$ then

$$
\int_{\{x: r(x)=t\}}\left|K_{\alpha}(x, y)\right|^{s} d \sigma(x) \asymp[r(y)+t]^{n-q}, n<q,
$$

and

$$
\sup _{w \in D}\left|K_{\alpha}(z, w)\right| \delta^{v}(z) \leqslant C \delta^{-\tilde{\alpha}+v}(z)
$$

$v \geqslant 0, v-\tilde{\alpha}<0$ and

$$
\int_{D}\left|K_{\alpha}(x, y)\right|^{s}(r(x))^{\beta} d v(x) \asymp(r(y))^{n-q+\beta+1}, n-q+\beta+1<0,
$$

and $r(y) \asymp \delta(y), y \in D ; q=\alpha s$.

Indeed using $(T)$ and mentioned estimates we have the following chain of inequalities

$$
\begin{gathered}
\prod_{j=1}^{k}\left(\int_{D}\left|f_{j}\left(w_{j}\right)\right|^{p} \delta^{\alpha_{j}}\left(w_{j}\right) d v\left(w_{j}\right)\right) \cdot \prod_{j=k+1}^{m} \sup _{\varepsilon>0} \int_{\partial D_{\varepsilon}}\left|f_{j}(\xi)\right|^{p} d \sigma(\xi)= \\
=C \prod_{j=1}^{k}\left\|f_{i}||_{A_{\alpha_{i}}^{p_{i}}}^{p_{i}} \cdot \prod_{j=k+1}^{m}\right\| f_{j} \|_{H^{p_{i}}}^{p_{i}} \leqslant \\
\leqslant C \int_{X} \int_{D} \prod_{j=1}^{m}\left|f_{s}(z)\right|^{p} \cdot\left|K_{s}\left(z, w_{j}\right)\right|^{p} \cdot \delta^{\beta p+(n+1)(p-1)}(z) d v(z) d \tilde{v}(x),
\end{gathered}
$$

where

$$
\begin{gathered}
s=\frac{\beta+n+1}{m} \\
\int_{X} d \tilde{v}(x)=\left(\prod_{j=1}^{k} \int_{D} \delta^{\alpha_{j}}\left(w_{j}\right) d v\left(w_{j}\right)\right) \cdot\left(\prod_{j=k+1}^{m} \sup _{\varepsilon>0} \int_{\partial D_{\varepsilon}} d \sigma(\xi)\right) .
\end{gathered}
$$

Applying Lemma 1 we have after small calculations that

$$
I \leqslant \tilde{C} \int_{D} \prod_{j=1}^{m}\left(\left|f_{j}(w)\right|^{p} \delta^{\tau}(w) d v(w)\right) .
$$

Theorem 1 is proved.

The proof of Theorem 2 is almost the same. We omit easy details.

Put

$$
B M O A_{t, v, s}^{p}=\left\{f \in H(B): \sup _{z \in B} \int_{B} \frac{|f(w)|^{p}(1-|w|)^{t}}{|1-\bar{z} w|^{v}} d v(w) \times(1-|z|)^{s}<\infty\right\},
$$


$v, s \geqslant 0, p>0, t>-1$.

For $p \geqslant 1$ this is a Banach space and complete metric space for $p<1$. Obviously based on properties of $r$-lattices (the same result with same proof is valid in pseudoconvex domains) based on vital estimate from below of Bergman Kernel on Bergman ball $($ see $[6,13])$,

$$
\|f\|_{B M O A_{t, v s}^{p}} \geqslant C \sup _{z \in B}|f(z)|(1-|z|)^{s+t-v+n+1},
$$

$v, s \geqslant 0, p>0, t>-1$.

This uniform estimate leads immediately to next theorem.

Theorem 3. Let $f_{i} \in A_{\alpha_{i}}^{p}, i=1, \ldots, m$ and $f_{j} \in B M O A_{t_{j}, s_{j}, v_{j}}^{p}, j=m+1, \ldots, m+k$.

Let $0<p \leqslant 1, s_{j} \geqslant 0$ and also $t_{j}>-1, v_{j} \geqslant 0, j=m+1, \ldots, m+k, \alpha_{k}>-1, k=1, \ldots, m$; let $v_{j}-s_{j}-t_{j}<n+1$,

$$
\frac{\beta+n+1}{m+1} p<t_{j}+n+1<\frac{\beta+n+1}{m+1} p+v_{j}-s_{j},
$$

$j=m+1, \ldots, m+k, \beta>\beta_{0}, n \in \mathbf{N}, m>1, m \in \mathbf{N}$.

Then for $\delta(z)=1-|z|, z \in B$, we have

$$
\int_{B} \prod_{j=1}^{m+k}\left|f_{j}(z)\right|^{p} \delta(z)^{\tau} d v(z) \asymp \prod_{k=1}^{m}\left\|f_{k}\right\|_{A_{\alpha_{k}}^{p}}^{p} \times \prod_{j=m+1}^{m+k}\left\|f_{j}\right\|_{B M O A_{t_{j}, s_{j}, v_{j}}^{p}}^{p}
$$

if

$$
\prod_{j=1}^{m+k} f_{j}\left(z_{j}\right)=C_{\beta} \int_{B} \prod_{j=1}^{m+k} f_{j}(w) \frac{1}{(1-z \bar{w})^{\frac{\beta+n+1}{m+k}}} \delta^{\beta}(w) d v(w),
$$

$\beta>\beta_{0}, z_{j} \in B, j=1, \ldots, m+k ; \beta_{0}$ is large enough

$$
\tau=(m-1)(n+1)+\sum_{k=1}^{m} \alpha_{k}+\sum_{j=m+1}^{m+k}\left(t_{j}+s_{j}-v_{j}\right)+(n+1) k .
$$

Remark 3. A third group with $\prod_{j=1}^{k}\left\|f_{j}\right\|_{H^{p}}^{p}$ can also be added in mentioned relation of Theorem 3 with similar proof. One part of theorem (estimate from above) can be even given with group of more general $\prod_{i=1}^{m}\left\|f_{i}\right\|_{A_{\alpha_{i}}^{p_{i}}}^{p_{i}}$ form with almost same proof.

Proof of Theorem 3.

Proof of Theorem 3 we have as in previous theorems. The proof is based on uniform estimate for BMOA we provided above, arguments of proof of previous theorem and the following Lemma.

Lemma A (See [21]). Let $s>-1, r, t \geqslant 0, r+t-s<n+1$ then

$$
\int_{B} \frac{(1-|z|)^{s} d v(z)}{|1-\bar{z} w|^{r}\left|1-\bar{z} w_{1}\right|^{t}} \leqslant \frac{\tilde{C}}{\left|1-w \bar{w}_{1}\right|^{r+t-s-n-1}}, \quad w, w_{1} \in B ; \quad r-s, t-s<n+1,
$$

for some constant $\tilde{C}>0$.

We omit easy details leaving some calculations with indexes to interested readers. Even more for other restriction to parameters this theorem is valid in bounded pseudoconvex domain with smooth boundary $D$. We'll discuss in other our papers this in more detail.

We will formulate that interesting result also below. The proof (in BMOA spaces in bounded pseudoconvex domains) is the same as in theorem above, but is based on new Lemma (see [17]). 
We will below provide proofs of unit ball case in $D$ the proof is practically the same.

Lemma B. Let $t-s<n+1<r-s, s>-1, r, t \geqslant 0, r+t-s>n+1$

$$
\int_{D}\left(\delta^{s}(z)\left|K_{r}(z, w)\right| \cdot\left|K_{t}\left(z, w_{1}\right)\right|\right) d v(z) \leqslant \frac{C}{(\delta(w))^{r-s-n-1}}\left|K_{t}\left(w, w_{1}\right)\right|,
$$

where $w, w_{1} \in D$.

The rest is the simple repetition of arguments of previous theorems.

We first consider model case of the unit ball. The proof of general case is the same. We have (when one BMOA is in chain) the general case with several functions from BMOA is the same.

$$
\left|\prod_{j=1}^{m+1} f_{j}\left(w_{j}\right)\right|^{p}=C_{\beta} \int_{B} \frac{\left|\prod_{j=1}^{m+1} f_{j}(z)\right|^{p} \times(1-|z|)^{\beta p+(n+1)(p-1)} d v(z)}{\left|\prod_{j=1}^{m+1} 1-\bar{z} w_{j}\right|^{\frac{\beta+n+1}{m+1}}} .
$$

Then using Lemma A and well-known Forell-Rudin type estimate (see [7])

where

$$
J \leqslant C_{1} \int_{X} \int_{B} \frac{\prod_{j=1}^{m+1}\left|f_{j}(z)\right|^{p} \times(1-|z|)^{\beta p+(n+1)(p-1)} d v(z)}{\prod_{j=1}^{m+1}\left|1-\bar{z} w_{j}\right|^{\frac{\beta+n+1}{m+1}}},
$$

$$
\int_{X}=\sup _{\tilde{w}} \int_{B} \ldots \int_{B} \prod_{j=1}^{m}\left(1-\left|w_{j}\right|\right)^{\alpha_{j}} d v\left(w_{j}\right) \times \frac{\left(1-\left|w_{m+1}\right|\right)^{t} \cdot(1-(\tilde{w}))^{s}}{\left|1-w_{m+1} \tilde{w}\right|^{v}} d v\left(w_{m+1}\right) .
$$

Hence

$$
J \leqslant C \int_{B} \prod_{j=1}^{m+1}\left|f_{j}(z)\right|^{p}(1-|z|)^{\tau} d v(z) ; \quad \tau=(1+n)(m-1)+\sum_{k=1}^{m} \alpha_{k}+s+t-v+n+1 ;
$$

as easy calculation with indexes shows.

Indeed, we have of our Theorem 3 that $k=\left(\frac{\beta+n+1}{m+1}\right) p$;

$$
\begin{aligned}
& \sup _{\tilde{w} \in B} \int_{B} \frac{(1-|w|)^{t} d v(w)(1-|\tilde{w}|)^{s}}{|1-w \tilde{w}|^{v}|1-z w|^{k}} \leqslant \sup _{w \tilde{\in} B} \int_{B} \frac{(1-|w|)^{t} d v(w)}{|1-w \tilde{w}|^{v-s} \cdot|1-z \bar{w}|^{k}} \leqslant \\
& \leqslant \sup _{w \in B} \frac{1}{|1-w z|^{v-s+k-t-(n+1)}} \leqslant \frac{\tilde{\tilde{C}}}{\left(1-|z|^{r}\right.} ; \quad r=v-s+k-t-(n+1),
\end{aligned}
$$

if $t>-1, v-s-t<n+1, k-t<n+1, v-s+k-t-(n+1)>0$.

This finished the proof of our theorem for the case of the unit ball.

Now we consider the case of pseudoconvex domains, the proof is a repetition of unit ball case so we again fix our attention to the unit ball case in $C^{n}$.

We have the following chain of estimates now based on Lemma from [6] (see also above). The only change for general $D$ pseudoconvex domain is to replace $(1-|z|)^{\alpha}$ be $\delta(z)$ and $\frac{1}{|1-z w|^{\alpha+n+1}}$ by $K_{\alpha}(z, w)$.

$$
J=\int_{X} \prod_{j=1}^{m}\left|f_{j}\right|^{p} \leqslant C \int_{X} \int_{B} \frac{\prod_{j=1}^{m}\left|f_{j}\right|^{p} \times(\delta(z))^{\beta p+(n+1)(p-1)}}{\prod_{j=1}^{m}\left|1-\bar{z} w_{j}\right|^{\frac{\beta+n+1}{m} p}} d v(z),
$$


where

$$
\int_{X}=\sup _{w \tilde{\epsilon} B} \int_{B} \ldots \int_{B} \frac{\prod_{j=1}^{m-1}\left(1-\left|w_{j}\right|\right)^{\alpha_{j}} \times\left(1-\left|w_{m}\right|\right)^{t} \cdot(1-|\tilde{w}|)^{s} d v\left(w_{j}\right)}{\left|1-\bar{w}_{m} \tilde{w}\right|^{v}} .
$$

Taking into account Lemma $B$ we obtain

$$
J \leqslant C \int_{B} \prod_{j=1}^{m}\left|f_{j}(z)\right|(1-|z|)^{\tau} d v(z),
$$

where $\tau$ was defined in our previous theorem.

Indeed, for $w_{1}, \ldots, w_{m-1}$ variables we must use Forelly-Rudin estimates

$$
\int_{B} \frac{\left(1-\left|w_{j}\right|\right)^{\alpha_{j}} d v\left(w_{j}\right)}{\left|1-z w_{j}\right|^{\frac{\beta+n+1}{m}}} \leqslant C \cdot(1-|z|)^{\alpha_{j}-\frac{\beta+n+1}{m}+n+1}, \quad z \in B .
$$

These estimates are valid also in bounded pseudoconvex domains (see $[6,13])$.

Then by Lemma B we have

$$
M=\sup _{\tilde{w} \in B}\left(\int_{B} \frac{\left(1-\left|w_{m}\right|\right)^{t}}{\left|1-\bar{w}_{m} \tilde{w}\right|^{v}\left|1-z w_{m}\right|^{\frac{\beta+n+1}{m} p}} d v\left(w_{m}\right)\right)(1-|\tilde{w}|)^{s} \leqslant \sup _{\tilde{w} \in B} \frac{C}{\left.|1-z \tilde{w}|^{v-s} \cdot|1-| z \mid\right)^{u}}
$$

for $v-s<n+1, \frac{\beta+n+1}{m} p-t>n+1,-s+v+\frac{\beta+n+1}{m} p-t-(n+1)>0$, where $u=\frac{\beta+n+1}{m} p-t-(n+1)$;

$$
M \leqslant \tilde{C}(1-|z|)^{-v+s-u} .
$$

Our last general Theorem is the following.

Let

$$
\left(B M O A_{\tau, v, s}^{p}\right)=\left\{f \in H(D): \sup _{w \in D} \int_{D}|f(z)|^{p} \delta(z)^{t} \cdot\left|K_{v}(z, w)\right| d v(z) \cdot\left(\delta^{s}(w)\right)<\infty\right\},
$$

$0<p<\infty, v>0, t>-1, s \geqslant 0$ is a BMOA-type space in a bounded pseudoconvex domain with smooth boundary in $C^{n}$. BMOA type spaces in such domains were studied in [17].

Theorem 4. Let $p \leqslant 1$, let $v_{j}-s_{j}<n+1, \frac{\beta+n+1}{m} p-t_{j}>n+1,-s_{j}+v_{j}+\frac{\beta+n+1}{m} p-$ $-t_{j}-(n+1)>0, j=m+1, \ldots, k, \beta>\beta_{0}, n \in \mathbf{N}, m>1, m \in \mathbf{N}$ and $\alpha_{j}>-1, j=1, \ldots, m$ then if $s_{j}>0, t_{j}>-1, v_{j}>0, j=m+1, \ldots, m+k$ then the assertion of previous theorem is valid if we replace $\frac{1}{(1-z w)^{\tau}}$ by $K_{\tau}(z, w)$ for $\tau>0$ in pseudoconvex domains for $B M O A_{\tau, v, s}^{p}(D)$ spaces and for Bergman $A_{\alpha}^{p}$ spaces in same type domains.

Hence each $\left(f_{j}\right)$ can be represented as $A_{\alpha}^{p}$ or BMOA atoms (see [7]) if $\prod_{i=1}^{m+k}\left|f_{i}\right|^{p} \in L_{u}^{1}$, for some parameter $u$ and $m, k \in \mathbb{N}$. These results again coincide for $m=1$ with known results on atomic decomposition of $A_{\alpha}^{p}$ Bergman class theorems (see [6]).

Remark 4. Let us stress in all these assertion is vital in main estimate to keep at least one component $\left\|f_{i}\right\|_{A_{\alpha_{i}}^{p_{i}}}$ (Bergman space component) in right side of the main estimates.

Concerning groups without $\prod_{i=1}^{m}\left\|f_{i}\right\|_{A_{\alpha_{i}}^{p_{i}}}$ like $\prod_{i=1}^{k}\left\|f_{i}\right\|_{H^{p}}^{p} \times \prod_{i=k+1}^{m}\left\|f_{i}\right\|_{B M O A}$ our methods don't work other approached here must be invented, based maybe on other integral representations. 
The $p>1$ case can be probably covered similarly we refer to [6] for "pure" $A_{\alpha}^{p}$ case with $\prod_{i=1}^{m}\left\|f_{i}\right\|_{A_{\alpha_{i}}^{p}}$ groups $(p>1)$ based purely on Holders inequality.

Our methods also covers cases when at least one component is our product is Herz-type spaces. This will be treated in our other papers, so we can similarly also consider the following products

$$
\prod_{i=1}^{m}\left\|f_{i}\right\|_{B_{\alpha j}^{p, q}} \times \prod_{i=m+1}^{N}\left\|f_{i}\right\|_{H^{p}}^{p} \text { or } \prod_{i=1}^{m}\left\|f_{i}\right\|_{\tilde{B}_{\alpha}^{p, q}}^{p} \times \prod_{i=m+1}^{N}\left\|f_{i}\right\|_{B M O A}^{p}
$$

with some restrictions on indexes

or

$$
\left.\prod_{i=1}^{m}\left\|f_{i}\right\|\right|_{B_{\alpha_{j}}^{p, q}} \times\left.\prod_{i=m+1}^{N}\left\|f_{i}\right\|\right|_{\tilde{A}_{\beta_{j}}^{p}} \text { or }\left.\prod_{j=1}^{m}\left\|f_{j}\right\|\right|_{\tilde{B}_{\alpha_{j}}^{p, q}} ^{p} \times \prod_{j=m+1}^{N}\left\|f_{j}\right\|{\tilde{\tilde{A}_{\beta_{j}}^{p}}}
$$

with some restrictions on indexes.

These cases will be considered in our other papers, though methods of this and those papers will be rather similar.

Note all results of this paper have direct analogues also in analytic spaces in unbounded tubular domains over symmetric cones. Proofs of such type results can be obtained by simple substitution of our estimates we used in our proofs in pseudoconvex domains to parallel known estimates in tube domains and on some parallel known related facts on Bergman represenation formula in tubular domains (see for example [22] and references there).

The only additional condition is on Bergman Kernel in tube domains over symmetric cones is Lemma $\mathrm{B}$, which is probably also valid.in tube domains also.

\section{References}

[1] H.Triebel, Theory of function spaces, Birkhauser, Basel, 2006.

[2] G.Dafni, Hardy spaces on some pseudoconvex domains, Geom. Anal., 9(1994), 273-316.

[3] J.Garnett, J.Latter, The atomic decomposition of Hardy spaces of several complex variables, Duke Math. Journ., 45(1978), 815-845.

[4] S.Grellier, M.Peloso, Decomposition theorems for Hardy spaces on convex domains of finite type, Illinois Journal of Mathematics, 46(2002), no. 1, 207-232.

[5] S.Li, R.Shamoyan, Complex Var. Elliptic Equ,. 54(2009), no. 12, 1151-1162. DOI: $10.1080 / 17476930903276167$

[6] R.F.Shamoyan, M.Arsenovic, On distance estimates and atomic decompositions, Bull. Korean Math. Soc., 52(2015), no. 1, 85-103.

[7] K.Zhu, Spaces of Holomorphic Functions in the Unit Ball, Springer-Verlag, New York, 2005.

[8] P.Ahern, R.Schneider, Holomorphic Lipschits functions in pseudoconvex domains, Amer. J. Math., 101(1979), no. 3, 543-565.

[9] R.Coifman, R.Rochberg, Representation theorems for holomorphic functions in $L^{p}$, Asterisque 77, Soc. Math. France, Paris, 1980, 11-66.

[10] Z.Chen, W.Quyang, Publicacions Matematiques, 52(2013), no. 2. DOI: 10.5565 /PUBLMAT_58214_18

[11] J.M.Ortega, J.Fabregs, Mixed-norm spaces and interpolations, Studia Math., 109(1994), no. $3,233-254$. 
[12] F.Beatrous, $L^{p}$-estimates for extensions of holomorphic functions, Michigan Math. Jour., 32(1985), no. 3, 361-380.

[13] M.Abate, J.Raissy, A.Saracco, Toeplitz operators and Carleson measure in strongly pseudoconvex domains, J. Func. Anal., 263(2012), no. 11, 3449-3491.

[14] R.Shamoyan, S.Li, On some estimates and Carleson type measure for multifunctional holomorphic spaces in the unit ball, Bull. Sci. Math., 134(2010), no. 2, 144-154.

[15] R.M.Range, Holomorphic Functions and Integral Representations in Several Complex Variables, Graduate Texts in Mathematics, Vol. 108, Springer-Verlag, New York, 1986.

[16] S-Y.Li, W.Luo Analysis on Besov spaces II. Embedding and duality theorems, J. Math. Anal. Appl., 333(2007), no. 2, 1189-1202.

[17] M.Andersson, H.Carlsson, $Q_{p}$ spaces in strictly pseudoconvex domains, Journal d'Analyse Mathematique, 84(2001), 335-359.

[18] J.Ortega, J.Fabrega, Pointwise multipliers and corona type decomposition in BMOA, Ann Inst. Fourier, 46(1996), no. 1, 111-137.

[19] W.S.Cohn, Weighted Bergman projections and tangential area integrals, Studia Math., 106(1993), no. 1, 59-76.

[20] E.Ligocka, On the Forelly-Rudin constructions and weighted Bergman projections, Studia Math., 94(1989), no. 3, 257-272.

[21] N.Kerzman, E.M.Stein, The Szego kernel in terms of Cauchy-Fantappie kernels, Duke Math. J., 45(1978), no. 2, 197-223.

[22] B.Sehba, Operators in some analytic function spaces and their dyadic counterparts, Glasgow, PhD, Dissertation, 2009.

\title{
О новых теоремах разложения в некоторых пространствах аналитических функций в ограниченных псевдовыпуклых областях
}

\author{
Роми Ф.Шамоян \\ Елена В. Томашевская \\ Брянский государственный университет \\ Брянск, Российская Федерация
}

\footnotetext{
Аннотация. Мы даем новые точные теоремы разложения для многофункциональных пространств Бергмана в единичном шаре и ограниченных псевдовыпуклых областей с гладкой границей, расширяющей известные результаты из единичного шара.

А именно мы докажем, что $\prod_{j=1}^{m}\left\|f_{j}\right\|_{X_{j}} \asymp\left\|f_{1} \ldots f_{m}\right\|_{A_{\alpha}^{p}}$ для различных $\left(X_{j}\right)$ пространства аналитических функций в ограниченных псевдовыпуклых областях с гладкой границей, где $f, f_{j}$, $j=1, \ldots, m-$ аналитические функции, а $A_{\alpha}^{p}, 0<p<\infty, \alpha>-1-$ пространство Бергмана. Это, в частности, также расширяет в разных направлениях известную теорему об атомном разложении пространств $A_{\alpha}^{p}$ Бергмана.

Ключевые слова: псевдовыпуклые области, единичный шар, пространства Бергмана, классы типа Харди, теоремы декомпозиции.
} 